\title{
Wettlauf gegen die Zeit: Ein handlungsorientierter Unterrichtseinstieg zum Wandel der Produktion
}

*a01509682@unet.univie.ac.at, Institut für Geographie und Regionalforschung, Universität Wien
*a01468971@unet.univie.ac.at, Institut für Geographie und Regionalforschung, Universität Wien
***a01402313@unet.univie.ac.at, Institut für Geographie und Regionalforschung, Universität Wien
***a01546731@unet.univie.ac.at, Institut für Geographie und Regionalforschung, Universität Wien

eingereicht am: 09.11.2018, akzeptiert am: 20.02.2019

Ein durchdachter Einstieg kann die Wirkung von Unterrichtseinheiten verstärken. In diesem Beitrag sollen die Typen, Funktionen und Kriterien guter Einstiege beleuchtet, ein praktisches Beispiel vorgestellt und dessen Effektivität theoretisch begründet werden. Dabei handelt es sich um ein Experiment, welches die Produktionsformen ,Industrielle Fließbandarbeit‘ und ,Manufakturarbeit‘ am Beispiel des Bastelns von Papier-Uhren veranschaulicht. Danach werden Anknüpfungsmöglichkeiten für den Unterricht behandelt und die erste praktische Anwendung reflektiert.

Keywords: Unterrichtseinstieg, Handlungsorientierung, Produktionsformen, Fließbandarbeit, Manufakturarbeit

\section{A race against the clock: an activity-oriented lead-in for the topic of economic transition}

A carefully prepared lead-in can increase the impact of a teaching unit. In this article, types and functions of and criteria for effective lead-ins will be examined. Furthermore, a practical example will be introduced, and its effectivity will be theoretically justified. The example consists of an experiment which illustrates the following forms of production: 'assembly line work' and 'factory work'. This is done by having the students produce clocks made of paper. In addition, follow-up activities as well as a reflection on the first application in the classroom will be presented.

Keywords: lead-in, activity orientation, forms of production, assembly line work, factory work

\section{$1 \quad$ Einleitung}

Bei der Unterrichtsvorbereitung wird die Planung eines thematisch bezogenen Stundeneinstiegs oft vernachlässigt. So schreibt Budke (vgl. 2013: 22), dass $89 \%$ aller Unterrichtseinstiege in der von ihr durchgeführten Studie lediglich aus einer Begrüßung bestünden. Eine derartige Stundeneröffnung hat jedoch keine didaktischen Funktionen für den weiteren Stundenverlauf, welche laut Budke im Idealfall aber vorhanden sein sollen.

Sie können sich vielleicht an Momente in Ihrer eigenen Schulkarriere erinnern, in denen Sie sich nach zwanzig Minuten der intensiven Beschäftigung mit einem Thema gefragt haben: „Worum geht es hier eigentlich?" Ein passender Einstieg kann solche Vorfälle verhindern, die darauffolgenden Schritte spannender, einfacher oder effizienter machen und den Schülerinnen und Schülern die Möglichkeit geben, sich auf das Kommende einzustimmen. Interesse zu wecken ist dabei zwar ein wichtiger Punkt und sollte bei Einstiegen immer mitgedacht werden, aber dieser Aspekt ist bzw. muss bei Weitem nicht die einzige Funktion eines guten Einstiegs sein.

Dieser Beitrag stellt Grundlegendes über Unterrichtseinstiege kurz dar und beschreibt ein Beispiel eines Einstieges aus der Praxis, welches mithilfe der theoretischen Konzepte von Meyer (2005) und Budke (2013) didaktisch begründet wird. Darauffolgend werden Anknüpfungsmöglichkeiten für den weiteren Stundenverlauf dargestellt und die Testung des Einstiegs in der Praxis reflektiert. 


\section{Relevante Theorien und theoretische Überlegungen}

Die Gestaltung eines nachhaltigen Stundeneinstiegs ist weniger trivial als gedacht. Es gilt sich erst einmal darüber klar zu werden, welche Funktionen dieser im Unterricht hat. Hilfreich für den weiteren Stundenverlauf, könnte ein Stundeneinstieg dazu verwendet werden, um ergänzend zur Begrüßung einen Einstieg in einen neuen Lernprozess einzuleiten und die Schüler/innen dazu zu bringen, motiviert und interessiert in einen neuen Themenblock zu starten. Um Einstiege effizient und wirkungsvoll planen und einsetzen zu können, ist es wichtig die Begrifflichkeiten vorerst zu definieren und möglichst genau abzugrenzen.

\subsection{Funktionen von Unterrichtseinstiegen}

Meyer (2005: 123) definiert den Unterrichtseinstieg folgendermaßen: „Der Unterrichtseinstieg soll - mit unmittelbarer oder mittelbarer Hilfe des Lehrers [sic!] - die Schüler [sic!] für das Thema und das Thema den Schülern [sic!] erschließen. "Ähnlich hierzu bezeichnet Klafki (1963 - Zitiert in: Ferrarÿ 2014: 9) den Einstieg als „Prozess der doppelseitigen Annäherung“. Laut Meyer (2005: 123) soll in einem Unterrichtseinstieg „das Thema so aufbereitet werden, daß [sic!] die Schüler [sic!] in den Stand versetzt sind, es möglichst selbsttätig anzueignen; andererseits sollen sich auch die Schüler [sic!] auf das Thema zubewegen [...]". Daher haben Lehrpersonen die Verantwortung die Lebenswelt der Schüler/innen anzusprechen und somit ihr Interesse zu wecken. Dabei steht auch das Ziel im Vordergrund, die Schüler/innen dort abzuholen, wo sie gerade sind. So kann auch von den Schülerinnen und Schülern erwartet werden, dass sie sich auf das Thema einlassen.

Die Anforderungen an Unterrichtseinstiege, von welchen manche bereits ansatzweise aufgegriffen wurden, sind in der nachfolgenden Tabelle zusammengefasst. Weiters wird im Folgenden auf die Kategorisierungen nach Meyer und Budke eingegangen:

Tab. 1: Anforderungen an Unterrichtseinstiege (vgl. Meyer 2005: 122)

\begin{tabular}{ll}
\hline Funktional & Themenbezogen \\
\hline Fragehaltung wecken & $\begin{array}{l}\text { Interesse \& Aufmerksamkeit } \\
\text { auf das neue Thema lenken } \\
\text { Vorkenntnisse \& Vorerfahrun- } \\
\text { gen anregen }\end{array}$ \\
$\begin{array}{l}\text { Beugierig machen } \\
\begin{array}{l}\text { Bestehende Vorstellungen } \\
\text { aufgreifen und hinterfragen } \\
\text { Information über geplanten } \\
\text { Stundenverlauf geben }\end{array}\end{array}$ & $\begin{array}{l}\text { Inhalten verknüpfen } \\
\text { Pon nen }\end{array}$ \\
\hline
\end{tabular}

Ein guter Unterrichtseinstieg zeichnet sich dadurch aus, möglichst vielen dieser funktionalen und themen- bezogenen Anforderungen zu entsprechen. Allen gerecht zu werden, wird allerdings in der Praxis ein eher unrealistisches Unterfangen darstellen. Nichtsdestotrotz wäre es für die Qualität des Einstiegs sinnvoll, diese Ansprüche in der Planung zu berücksichtigen. An dieser Stelle muss auch betont werden, dass der Einsatz dieser Funktionen nicht automatisch zu einem gelungenen Unterrichtseinstieg führt. Dies wird besonders durch die unvorhersehbaren Bedingungen sowie durch die Reaktionen, welche in der Praxis aufkommen, unterstrichen. So ist es in der Tat möglich einen Unterrichtseinstieg bis in das kleinste Detail zu planen. In der Realität agieren Personen jedoch situationsbezogen, wodurch selbst ein ausgezeichnetes Einstiegskonzept in eine etwas andere Richtung verlaufen kann. (vgl. Meyer 2005: 129)

Meyer (2005: 129) weist auf die eminenten Unterschiede „zwischen den in der Theorie entwickelten didaktisch-methodischen Ansprüchen und den in der Praxis vorgefundenen Arbeitsbedingungen" hin. Auf Grund dessen formuliert er didaktische Kriterien für gute Unterrichteinstiege, auf denen in der Planung aufgebaut werden kann. Wichtig ist, dass die von ihm definierten Kriterien als Anhaltspunkte für die Praxis dienen und nicht als eine Anordnung die strikt zu befolgen ist. Die fünf didaktischen Kriterien von Meyer für „die Planung und Beurteilung" von Unterrichtseinstiegen sollen im Folgenden erläutert werden.

Didaktische Kriterien nach Meyer (vgl. 2005: 129)

1. Orientierungsrahmen

2. Zentrale Aspekte

3. Vorverständnis

4. Disziplinierung

5. Handelnder Umgang mit dem neuen Thema

\section{Einen Orientierungsrahmen schaffen}

Ziel des Orientierungsrahmens ist es, den Schülerinnen und Schülern einen Überblick über das Kommende zu geben. Die Schüler/innen sollen sich so auf den Umfang und die Aspekte des Themas einstellen und gegebenenfalls mitplanen. Das Einbeziehen der Schüler/innen ist deshalb wichtig, da diese Phase des Unterrichts aufgrund der vorangehenden lehrer/innenzentrierten Planung oft anfänglich keine aktive Schüler/innenrolle fordert. Deshalb sollte die Orientierungsphase - wann auch immer möglich - einer schüler/innenzentrierten und handlungsorientierten Einführung dienen. (vgl. Meyer 2005: 130 f.)

\section{Zum Kern der Sache kommen}

Eine wichtige Funktion des Einstiegs ist es, anfänglich die relevanten Punkte des Themas aufzuzeigen, die im Anschluss behandelt werden. Meyer (2005: 131) be- 
schreibt den Einstieg als eine „Schlüsselszene“. Ferrarÿ (2014: 12) betont, „dass Aufwand und Nutzen in einem realistischen Verhältnis stehen sollten “. Es ist daher darauf zu achten, dass neben dem Einstieg auch noch genug Zeit für Erarbeitungs-, Übungs- und Reflexionsphasen bleibt.

Aktivierung des Vorverständnisses der Schüler/innen Im Wissenschaftsbereich der Pädagogik wird der Begriff des „Vorwissens" häufig unterschiedlich definiert. Meyer (2005: 132) beschreibt den Begriff als „das Gesamt an Vorkenntnissen, Einstellungen, Interessenslagen und Haltungen der Schüler [sic!] [...], die ihr Denken, Fühlen und Handeln im Unterricht steuern". In der Praxis stehen vor allem die Wissensverknüpfung, die Interessensweckung und das Aufbrechen von Denkmustern im Fokus.

\section{Eine disziplinierte Arbeitshaltung herstellen}

Mit der Disziplinierungsfunktion will Meyer (vgl. 2005: 133) nicht auf eine extrinsische Disziplinierung der Schüler/innen hinaus, sondern darauf, dass die Lehrperson mittels eines durchdachten Einstieges eine gewisse Haltung ihrer Schüler/innen dem Thema gegenüber erzeugt. Im Unterricht würde sich dies etwa durch konkrete themenbezogene Fragen seitens der Schüler/innen äußern.

\section{Einen handelnden Umgang mit dem neuen Thema ermöglichen}

Der letzte Punkt, der in dieser Auswahl als relevant erscheint, thematisiert die Handlungsorientierung von Unterrichtseinstiegen. Diese Art und Weise des Einstiegs soll die Schüler/innen zum selbstständigen Agieren anregen. Meyer (2005: 133) betont die Wichtigkeit des „Selbst-Erproben-Können“. Dadurch ist potentiell eine größere Anzahl an Schülerinnen und Schülern aktiv in den Unterricht involviert.

Ergänzend zu den Ansprüchen und den didaktischen Kriterien ist eine grobe Klassifizierung von Einstigen sinnvoll. Eine nennenswerte Klassifizierung für viele Unterrichtseinstiege sind die Problemtypen nach Budke (2013: 24) für „problemorientierte Unterrichtseinstiege". Nicht alle Unterrichtseinstiege erfüllen das Kriterium der Problemorientiertheit, es erscheint uns dennoch wichtig hier kurz darauf hinzuweisen, da die zugehörigen Problemtypen eine gute Grundlage für die Methodenfindung darstellen. Die Autorin (2013: 23) nimmt dabei eine Einteilung "nach der Art des zu thematisierenden Problems" vor und unterscheidet folgende Problemtypen (vgl. Budke 2013: $23 \mathrm{ff})$ :

1. $\quad$ Einstieg über Wissenslücken

2. Einstieg über Widersprüche

3. Einstieg über Kompliziertheit
Bei Ersterem geht es um das Bewusstmachen von Wissenslücken, die durch die Untersuchung und Beantwortung von aufgeworfenen Fragen beseitigt werden sollen.

Der Einstieg über Widersprüche soll Schüler/innen in die Lage versetzen, eine Fragestellung auf Grund von widersprüchlichen Informationen nicht beurteilen zu können. Die Lehrperson konfrontiert dazu die Jugendlichen mit ihren Vorkenntnissen und Voreinstellung widersprechenden Informationen. Dabei soll auch die eigene Konstruktion der Wirklichkeit hinterfragt werden. Außerdem kann ein Ziel des Einstiegs über Widersprüche auch das Verstehen und Nachvollziehen von unterschiedlichen Sichtweisen verschiedener Akteurinnen und Akteure zu einem bestimmten Thema sein. Aus diesem Verständnis kann dann die eigene Meinung gebildet werden.

Zuletzt nennt Budke den Einstieg über die Kompliziertheit. Er bietet sich bei Themen an zu denen die Schüler/innen schon viel Wissen haben, was meist gegen Ende eines Themenblocks der Fall ist. Bei diesem Einstiegstyp muss den Lernenden die Kompliziertheit ihrer Sicht bewusst werden, um ein Problem zu lösen.

Diese Typen können als Orientierungsrahmen dienen und die Auswahl der passenden Methoden erleichtern. In Tabelle 2 soll ein grober Überblick über die Typen und eine Auswahl passender Einstiegsmethoden gegeben werden.

Tab. 2: Methoden nach Einstiegstypen (ergänzt von Pichler 2018 nach Budke 2007)

\begin{tabular}{|c|c|}
\hline Einstiegstypen & Einstiegsmethoden (Auswahl) \\
\hline Einstieg über Wissenslücken & $\begin{array}{l}\text { Demonstration, Filmausschnitt, Si- } \\
\text { mulation, Kontraste, Spurensuche, } \\
\text { Exkursion, Fantasiereise, etc. }\end{array}$ \\
\hline Einstieg über Widersprüche & $\begin{array}{l}\text { Interessenkonflikte im Rollenspiel, } \\
\text { Karikaturen, Interviews, Pro- und } \\
\text { Kontrakommentare, etc. }\end{array}$ \\
\hline Einstieg über Komplexität & $\begin{array}{l}\text { Partnerinterview, Rollenspiel, } \\
\text { Bilder, etc. }\end{array}$ \\
\hline
\end{tabular}

\subsection{Theoretische Begründung des Beispielein- stiegs - Basteln von Papieruhren}

Die Vielzahl an theoretischen Ansätzen über Unterrichtseinstiege macht es notwendig unseren Praxisvorschlag in diese Theorien einzuordnen und zu zeigen, welche Kriterien ihn zu einem erfolgreichen Unterrichtseinstieg machen können.

Das hier vorgestellte Experiment lässt sich in die Problemkategorie "Wissenslücke“ nach Budke (vgl. 2013: 25) einordnen. Der Einstieg adressiert die Wissenslücke, dass sich die Schüler/innen unter den beiden industriellen Produktionsformen ,Fließbandarbeit' und ,Manufakturarbeit' aufgrund mangelnder 
beruflicher Erfahrung vermutlich nicht viel vorstellen können. Durch das eigene Ausüben von Tätigkeiten in der ,Manufaktur' beziehungsweise am ,Fließband" wird diese Wissenslücke bewusst angesprochen. Auf Ebene des Unterrichts geht es also um das Klären eines Verständnisproblems (vgl. Budke: 24f). Im weiterführenden Unterricht können damit eine Vielzahl an unterschiedlichen Aspekten der beiden Arbeitsund Produktionsformen gegenübergestellt und diskutiert werden.

Wie bereits erwähnt ist eine Bewusstmachung dessen, welche didaktischen Kriterien der geplante Einstieg erfüllt, wichtig für die Qualitätssicherung. Dem Einstieg können folgende Funktionen nach Meyer (vgl. 2005: 129) zugeordnet werden:

1. Einführung in zentrale Aspekte des Themas anhand einer exemplarischen Darstellung (die modellhafte Durchführung der Produktionsformen)

2. Anknüpfen an Vorverständnis (Produktionsprozesse, die die Schüler/innen in diesem Fall selbst ausprobieren und mit Vorerfahrung und Vorwissen vergleichen können)

3. Herstellung einer disziplinierten Arbeitshaltung (die Schüler/innen haben die Möglichkeit mitzuwirken, dies erhöht den Motivationsfaktor)

4. Handlungsorientierung (die Schüler/innen simulieren einen Produktionsvorgang und sind somit selbst produktiv; die haptische Wahrnehmung sowie Emotionen werden angesprochen; alle tragen zum Gelingen des Experiments bei)

Die Handlungsorientierung steht im Falle des von uns kreierten Einstiegsexperiments klar im Vordergrund. Durch das eigene Handeln nehmen die Schüler/innen die Emotionen und Charakteristika, die mit den jeweiligen Produktionsformen verknüpft sind, durch ihre eigenen Erfahrungen mit und können sich selbst in die Situation einer Arbeitenden/eines Arbeitenden versetzen. So wird auch die zuvor angesprochene Wissenslücke langsam geschlossen. Durch das direkte Einbinden aller Schüler/innen ist der Einstieg außerdem schüler/innenzentrierter. Ein weiterer Vorteil besteht darin, dass von diesem Experiment sehr einfach in das kritisch-emanzipatorische Vermittlungsinteresse übergeführt werden kann, indem zum Beispiel Arbeits- und Produktionsbedingungen hinterfragt werden. Aber auch auf Ebene des technischen Vermittlungsinteresses („Wie funktionieren industrielle Produktionsabläufe?"), sowie des praktischen Vermittlungsinteresses („Was bedeutet dies für die Arbeitsbedingungen in zukünftigen Arbeitsfeldern, die für mich als Berufe in Frage kommen?") kann weitergearbeitet werden.

Rückblickend auf die zu Beginn genannten Anforderungen an Unterrichtseinstiege (vgl. Meyer 2005: 122) oder auch auf die didaktischen Kriterien (vgl. Meyer 2005: 129), könnte man anmerken, dass die Anforderung, über den weiteren Stundenverlauf $\mathrm{zu}$ informieren' in diesem Einstieg nicht aufgegriffen wird. Vor dem Experiment will man schließlich nicht zu viel vorwegnehmen und eine gewisse Neugierde erhalten. Dieser informierende, organisatorische Aspekt könnte jedoch später nachgeholt werden.

Insgesamt kann festgehalten werden, dass der Einstieg die Schüler/innen auf das Kommende einstimmen und eine motivierte, interessierte Arbeitshaltung schaffen soll. In der Unterrichtspraxis sollten die ausgewählten Einstiege jedoch gut durchdacht und mit einem Ziel für die Unterrichtseinheit verknüpft werden. Wird hingegen nur ein kurzer fesselnder Moment geplant, so kann es vorkommen, dass der Ehrgeiz und das Interesse der Schüler/innen, einem Strohfeuer gleich, verpuffen. In dem hier präsentierten Beispiel wäre das Unterrichtsziel den Lernenden die Unterschiede zweier Produktionsformen, Fließbandarbeit und Manufaktur, zu veranschaulichen.

Zusammenfassend lässt sich feststellen, dass Einstiege einen wesentlichen Einfluss auf den Erfolg des nachfolgenden Unterrichts haben und somit die Thematik der Unterrichtseinstiege eine äußerst wichtige ist, da sie den Grundstein für nachfolgende Unterrichtsschritte legt. Auch wenn man bedenken muss, dass nach dem Einstieg noch ausreichend Zeit für anschließende Unterrichtsphasen bleiben muss, sollten passende Gelegenheiten für zeitaufwendigere Einstiege, wie in Punkt 3 praktisch dargestellt, nicht verabsäumt werden.

\section{Der Einstieg: Vorbereitung, Organisation und Ablauf}

Das folgende Experiment soll in der 7. Schulstufe als Unterrichtseinstieg in die Thematik der Arbeitswelt dienen und lässt sich im Lehrplan unter „Einblicke in die Arbeitswelt“ insbesondere beim Punkt „Den stetigen Wandel der Arbeitswelt erkennen und daraus die Einsicht in die Notwendigkeit der ständigen Weiterbildung und Mobilität gewinnen" einordnen (BMBWF 2000: 4). Die Schüler/innen werden bei diesem Experiment Uhren aus Papier produzieren. Ziel des Experimentes ist, den Lernenden die Unterschiede zweier Produktionsformen, Fließbandarbeit und Manufaktur, näherzubringen. Ähnliche Experimente gibt es zwar bereits zum Beispiel mit Papierfliegern (Klappacher et al. 2014: 74), allerdings eignet sich die Uhr insbesondere als Produkt, weil sie einen symbolischen Wert im Zusammenhang mit dem Zeitdruck in der Fließbandarbeit hat. Zudem haben wir es uns zur Aufgabe gemacht, das Experiment detailreich zu erläutern und Ideen für die Nachbereitung anzubieten. 
Auch wenn die Vorbereitung für diesen Unterrichtseinstieg für Lehrpersonen einen größeren Arbeitsaufwand erfordert, macht er sich aufgrund der aktiven Tätigkeit und der erlebten Emotionen der Lernenden, sowie durch die vielseitigen daraus zu gewinnenden Erkenntnisse und Möglichkeiten die er in der Nachbereitung bietet, bezahlt.

\subsection{Vorbereitung}

\subsubsection{Zeitliche Übersicht}

Tab. 3: Zeitliche Übersicht

\begin{tabular}{ll}
\hline Unterrichtsphase & Dauer \\
\hline Organisation der Tische und Einteilung der Gruppen & $3 \mathrm{Min}$. \\
Erklärung des Experiments & $4 \mathrm{Min}$. \\
Durchführung des Experiments & $8 \mathrm{Min}$. \\
Aufräumen & $2 \mathrm{Min}$. \\
Nachbereitung in Verschnitt-Gruppen & $5 \mathrm{Min}$. \\
Nachbereitung im Plenum + Hefteintrag & $10 \mathrm{Min}$. \\
\hline
\end{tabular}

\subsubsection{Material}

Für das Experiment werden folgende Utensilien benötigt:

Gruppe 1: Uhren-Vorlagen, Scheren, spitze Bleistifte oder Zahnstocher, Filzstifte oder Kugelschreiber, Spreizklammern, Box für die fertigen Uhren

Gruppe 2: Scheren, Buntstifte, Filzstifte, Kugelschreiber, Lineale, Papier, Spreizklammern, Zirkel, Box für die fertigen Uhren

\subsection{Organisation}

Die Klasse wird in zwei gleich große Gruppen geteilt. Die Tische werden für Gruppe 1 nebeneinander angeordnet, sodass diese eine längliche Form bilden. Für Gruppe 2 werden die Tische so zusammengeschoben, dass die Schüler/innen rundherum Platz nehmen können. Es sollte im Raum möglichst viel Abstand zwischen den beiden Gruppen sein, damit sich diese während dem Experiment nicht gegenseitig hören oder ablenken.

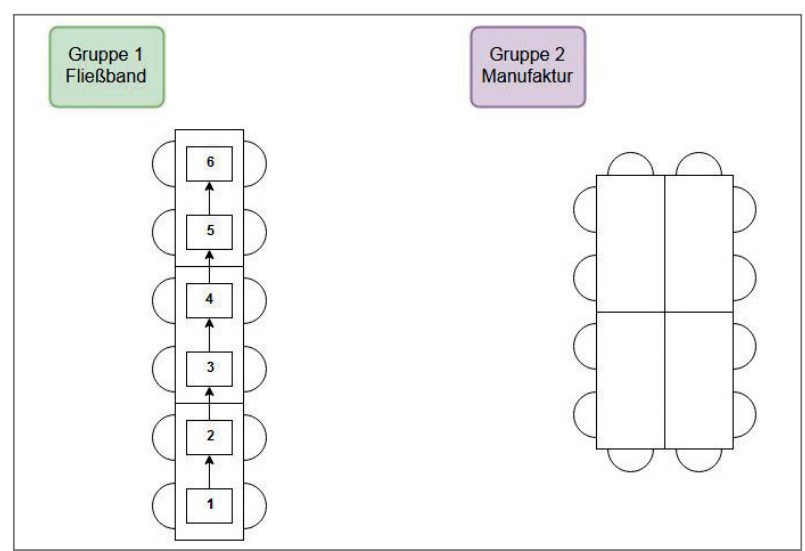

Abb. 1: Schema der Organisation der Klasse

\subsection{Erklärung und Durchführung}

Die Aufgabenstellung wird für beide Gruppen separat erklärt, damit die jeweilige Arbeitsweise nicht beeinflusst wird. Wenn zwei Lehrpersonen unterrichten, kann sich jeweils eine Lehrperson einer Gruppe widmen. Sollte man das Experiment als Einzellehrperson durchführen, werden die Gruppen hintereinander instruiert. Es kann zudem jede Teilaufgabe der Arbeitsauftrag in schriftlicher Form (als Rollenkarte) zur Verfügung gestellt werden.

Gruppe 1 ist die Gruppe, die am Fließband arbeiten wird. Es übernehmen jeweils zwei oder mehr (je nach Klassengröße) Schüler/innen, die sich gegenübersitzen, denselben Arbeitsschritt. Ausführliche und präzise Erklärungen sind hier erforderlich. Die einzelnen Arbeitsschritte werden im Folgenden erläutert:

1. Deine Aufgabe ist es, mithilfe der Uhrenschablone (siehe Schablone 1) einen Kreis auf ein Blatt Papier zu zeichnen. Markiere anschließend außerhalb des Kreises die Stellen, an denen die Ziffern eingezeichnet werden sollen. Sobald du fertig bist, reiche das Blatt Papier an Station 2 weiter.

2. Deine Aufgabe ist es, die Ziffern auf das Ziffernblatt zu schreiben. Reiche das Blatt anschließend an Station 3 weiter.

3. Bei dieser Station sollst du die Uhr ausschneiden. Reiche die ausgeschnittene Uhr gleich zu Station 6 weiter.

4. Deine Aufgabe ist es, die Minutenzeiger (siehe Schablone 2) auszuschneiden. Reiche deine fertigen Zeiger an Station 6 weiter.

5. Deine Aufgabe ist es, die Stundenzeiger (siehe Schablone 3) auszuschneiden. Reiche deine fertigen Zeiger an Station 6 weiter.

6. Bei dieser Station steckst du mithilfe der Spreizklammer die beiden Zeiger mit dem Ziffernblatt zusammen. Damit du dir beim Durchstecken leichter tust, stich die benötigen Löcher mit einem spitzen Bleistift oder Zahnstocher vor. Die fertigen Uhren werden in einer Box gesammelt.

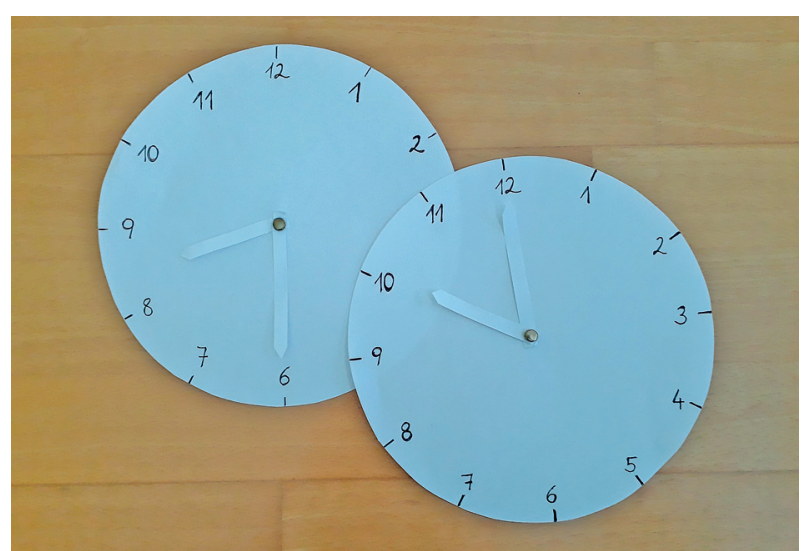

Abb. 2: Die Uhren der Fließband-Gruppe (eigene Darstellung) 
Außerdem wird der Gruppe mitgeteilt, dass sie schnell arbeiten muss, um möglichst viele Uhren zu produzieren. Falls bei einzelnen Stationen schneller gearbeitet wird als bei anderen, kann während des Experiments eventuell noch eingegriffen und eine Schülerin/ein Schüler aufgefordert werden, die Aufgabe zu wechseln und bei einem anderen Arbeitsschritt mitzuhelfen.

Gruppe 2 ist in ihrer Arbeitsweise deutlich freier. Sie erhält folgende Instruktionen: Eure Aufgabe ist es, mithilfe der Materialien (siehe oben) Uhren zu gestalten. Wie diese aussehen, entscheidet ihr. Sie können also angemalt und verziert werden und müssen auch nicht rund sein. Seid also kreativ. Die fertigen Uhren sammelt ihr in einer Box.

Beide Gruppen werden über die verfügbare Zeit informiert - wir empfehlen etwa acht Minuten. Der Zeitrahmen ist damit so gewählt, dass die Mitglieder der Gruppe 1 mehrere Uhren produzieren können und auch in Gruppe 2 manche Schüler/innen eine Uhr fertigstellen werden. Außerdem sollten die Mitglieder von Gruppe 1 gegen Ende der acht Minuten durchaus eine gewisse Langeweile beziehungsweise Anstrengung verspüren; schließlich geht es auch um die erlebten Emotionen, welche der Realität monotoner Fließbandarbeit nahekommen sollen. Am besten wird eine Stoppuhr mit dem Beamer an die Wand projiziert, sodass alle über die verbleibende Zeit Bescheid wissen. Kurz bevor die Zeit abläuft kann man die Schüler/innen gegebenenfalls noch über die verbleibenden Minuten bzw. Sekunden informieren. Mit Ablauf der Zeit wird das Experiment beendet. Die Schüler/innen werden aufgefordert ihre Utensilien nun beiseite zu legen, die Tische in ihre ursprüngliche Position zu bringen und Bastelabfälle wegzuräumen.

\subsection{Nachbereitung}

Die Uhren werden von der Lehrperson/den Lehrpersonen eingesammelt, gezählt und der Klasse gezeigt. Hier können die Schüler/innen erstens den Unterschied im Aussehen der Uhren identifizieren - die Uhren der Manufaktur-Gruppe werden wahrscheinlich variantenreicher und eventuell detailreicher gestaltet sein als die Uhren der Fließband-Gruppe. Zweitens können Unterschiede in der Anzahl der Uhren pro Gruppe festgestellt werden; die Fließband-Gruppe sollte deutlich mehr Uhren produziert haben. Anschließend werden die Lernenden in Vierergruppen eingeteilt, wobei immer zwei Mitglieder von Gruppe 1 und zwei Mitglieder von Gruppe 2 zusammenarbeiten. In den neu gebildeten Verschnitt-Gruppen bekommen die Lernenden die Aufgabe, sich gegenseitig von der verrichteten Arbeit zu berichten, um etwaige Unterschiede festzustellen.
Nach dieser Phase wird das Besprochene im Plenum gemeinsam mit der Lehrperson beziehungsweise den Lehrpersonen präsentiert und reflektiert. Die Lehrperson kann den Schülerinnen und Schülern mit unterschiedlichen Fragen helfen, deren Erfahrungen und Emotionen während der Arbeitsphase wiederzugeben und sie anleiten, Vermutungen über die beiden Produktionsformen anzustellen:

1. War euch während der Arbeit langweilig oder hat sie Spaß gemacht?

2. War die Arbeit eher eintönig oder abwechslungsreich?

3. Wie würdet ihr euch fühlen, wenn ihr diese Art von Arbeit länger machen müsstet?

4. Wenn ihr euch vorstellt dieser Arbeit über mehrere Jahre nachzugehen, welche (gesundheitlichen) Herausforderungen könnten dabei auftreten?

5. Habt ihr mehrere Arbeitsschritte durchgeführt oder nur einen?

6. Wo gab es klare Vorgaben und wo spielte Kreativität eine große Rolle?

7. Wo werden mehr Produkte hergestellt und wieso?

8. Welches Produkt kann im Anschluss warum teurer verkauft werden?

9. Bei welcher Produktionsform kann man sich eher mit dem Endprodukt identifizieren?

10. Bei welcher Produktionsform werden vermutlich mehr Mitarbeiter/innen angestellt sein? (Hier sollte in der weiteren Verarbeitung angesprochen werden, dass einfache Fließbandprozesse längst von Maschinen übernommen worden sind (Mechanisierung))

Diese Fragen können, je nach den weiterführenden Unterrichtszielen, angepasst werden.

In Stichworten sollen die Charakteristika der beiden Produktionsformen schließlich an der Tafel in zwei Spalten gegenübergestellt werden, sodass diese von den Schülerinnen und Schülern auch in ihre Hefte übertragen werden können.

\section{$4 \quad$ Anknüpfungsmöglichkeiten}

Wie zuvor bereits beschrieben, handelt es sich um ein Experiment das als Unterrichtseinstieg in die Thematik der ,Erkundung der Arbeitswelt' dient. Im Folgenden soll eine Möglichkeit aufgezeigt werden, wie an die eben beschriebene Nachbereitung angeschlossen werden kann. In dieser wurden bereits die Unterschiede der zwei Produktionsformen, Fließbandarbeit und Manufaktur, behandelt. Ganz unter dem Motto ,Zeitreise' erkundet man gemeinsam mit den Schülerinnen und Schülern den Wandel der Arbeitswelt. Aufbauend auf der historischen Entwicklung und der Analyse aktueller Arbeitsbedingungen, gilt es die Veränderungen 
der Arbeitswelt zu reflektieren, um auf zukünftige Jobs der Jugendlichen aufmerksam zu machen (z. B.: Digitalisierung).

Zunächst bietet es sich an, die beiden analysierten Produktionsformen zeitlich einzuordnen. Bei der Manufaktur handelt es sich um eine typische vorindustrielle Produktionsform. Abgelöst von der, durch das Fließband repräsentierten, industriellen Produktionsweise, konnte plötzlich eine Vielzahl mehr an Uhren oder ähnlichen Konsumgütern hergestellt werden. In diesem Zusammenhang können auch Standortverlagerungen thematisiert werden.

Die Industrie 4.0 stellt einen weiteren Wandel im Produktionssektor dar. Dabei steht „eine hochautomatisierte und vernetzte industrielle Produktions- und Logistikkette" im Vordergrund (BMAS 2015: 15). Doch wie genau mag die Produktion diverser Produkte im Zeitalter der so oft erwähnten ,Industrie 4.0 aussehen? Wie könnten Uhren im Jahr 2050 hergestellt werden?

Für letztere Frage schlagen wir einen Lernschritt vor, den man als Szenario-Technik bezeichnen könnte. Dabei sollen die Schüler/innen in Kleingruppen von vier Personen Zukunftsszenarien miteinander vergleichen. Ziel ist es, abzuwägen und sich auf ein Szenario zu einigen, das die Kleingruppe für am wahrscheinlichsten hält. Weiters sollen sie Argumente finden, die ihre Auswahl begründen. Es stehen folgende Szenarien zur Wahl:

1. In Zukunft werden Uhren innerhalb eines vollautomatischen Produktionssystems erzeugt. Jene Jobs, die dabei noch gebraucht werden, beziehen sich vor allem auf den Bau, die Installation und die Wartung von Produktionsrobotern. Hinzu kommen Jobs im Bereich Management und verschiedene Dienstleistungen.

2. Dank 3D-Druckern müssen in Zukunft lediglich die dafür notwendigen Modelle virtuell erstellt werden. IT-Expertinnen und -Experten sowie Grafiker/innen werden in diesem Szenario besonders am Arbeitsmarkt nachgefragt.

3. Ein gesellschaftliches Umdenken hat stattgefunden. Da mangelnde Beschäftigung und fehlende sinnstiftende Arbeit zu enormen sozialen und wirtschaftlichen Spannungen geführt haben, kehrt man zurück zur semi-manuellen Produktion, in welcher nachhaltige regionale Materialien und Produktindividualität eine besondere Bedeutung zukommt.

4. Keine der hier skizzierten Szenarien erscheint uns für wahrscheinlich. Wir denken die Uhrenerzeugung wird in Zukunft wie folgt aussehen: ...

In einer zirka zehnminütigen Phase einigen sich die Schüler/innen auf eines der Szenarien und begründen ihre Auswahl. Danach stellen sie diese im Plenum vor.
Da man sich kaum vorstellen kann, wie die Arbeitswelt, beziehungsweise, im Speziellen, die Uhrenproduktion in Zukunft aussehen wird, gibt es dabei keine ,richtige' oder ,falsche' Lösung. Das Hauptaugenmerk dieser Aufgabe soll auf der Erkenntnis liegen, dass solche Entwicklungen sich in den jeweiligen Segmenten des Arbeitsmarktes sehr unterschiedlich auswirken und, dass die Jobchancen in einer veränderten Arbeitswelt sehr häufig von den Qualifikationen der Arbeitnehmer/innen abhängen. Beispielsweise werden in einer automatisierten Produktionshalle gering qualifizierte Hilfsarbeiter/innen weniger gebraucht als Techniker/innen für die Maschinenprogrammierung. Besonders wichtig ist es die Schüler/innen darauf aufmerksam zu machen, dass sich unsere Arbeitswelt stetig wandelt und damit auch die Anforderungen des Arbeitsmarktes. Um diesen gerecht zu werden, ist lebenslanges Lernen essenziell.

\section{$5 \quad$ Reflexion und Fazit}

Während der ersten Durchführung des Experiments in einer 3. Klasse Unterstufe im Gymnasium wurde deutlich, dass der Einstieg die von uns gedachten Kriterien tatsächlich erfüllt. Der Einstieg war als exemplarische Darstellung von Produktionsformen gedacht, was einigen Schüler/innen bei der Nachbesprechung schnell auffiel und auch der Begriff, ,Fließbandarbeit wurde schnell genannt. Die Schüler/innen hatten im Folgenden keine Schwierigkeiten, das Erlebte als Beispiel eines ,echten' Produktionsprozesses zu erkennen, denn es wurden praxisnahe Vergleiche angestellt und auch Kritik am Herstellungsprozess geäußert. Beispielsweise begann ein Schüler der Gruppe 1 nach zwei Minuten den Herstellungsprozess zu optimieren, indem er die Sitzordnung und Aufgabenverteilung anpasste, und er thematisierte dies auch in der Nachbesprechung.

Weiters aktivierte bzw. knüpfte der Einstieg an das Vorwissen der Schüler/innen an. Begriffe wie Massenproduktion oder Konzepte wie ,Made in China' wurden bei der Nachbesprechung genannt und sogleich mit dem Experiment verknüpft oder vor dessen Hintergrund reflektiert.

Die große Stärke des Einstiegs ist die Handlungsorientierung. Die Möglichkeit zur aktiven Mitgestaltung wurde sogleich von einigen genutzt, wie am Beispiel des Versuchs zur Optimierung des Produktionsprozesses deutlich wird. Dadurch, dass jede einzelne Schülerin und jeder einzelne Schüler zum Handeln aufgefordert und die aktive Mitwirkung zum Gelingen des Experiments benötigt wurde, war das allgemeine Motivations- und Konzentrationsniveau sehr hoch. Auch für die eher frontal geführte Nachbesprechung 
hielt dieses Motivationslevel an. Handlungsorientierte Einstiege können somit auch eine positive Wirkung auf nachfolgende Unterrichtsphasen haben.

Die Handlungsorientierung führte außerdem dazu, dass es durch den Einstieg gelang, eine disziplinierte Arbeitshaltung herzustellen. Während des Experiments und auch in der darauffolgenden Phase waren die Schüler/innen sehr aufmerksam und auf die Arbeit fokussiert; manche gingen völlig in ihrer Tätigkeit auf.

Der Einstieg eignet sich besonders für TeamTeaching-Situationen, da die Vorbereitung und die Betreuung der Gruppen aufgeteilt werden kann. Mit entsprechender Planung und Adaptierung (z. B. Instruktionen für Gruppe 2 schriftlich) kann der Einstieg auch von einer alleine agierenden Lehrperson durchgeführt werden. Die benötigte Unterrichtszeit von etwa 30 Minuten, nur für den Einstieg, zahlte sich retrospektiv betrachtet aus, da sich die Nachbereitung sehr ergiebig und effizient gestaltete und somit insgesamt relativ rasch in komplexere Phasen übergegangen werden konnte.

Somit möchten wir von unserer Erfahrung ausgehend andere Lehrer/innen ermutigen, diesen und andere handlungsorientierte Unterrichtseinstiege auszuprobieren und in ihr Repertoire aufzunehmen.

\section{Literatur}

BMAS (Hrsg.) (2015): Grünbuch Arbeiten 4.0. https:// www.bmas.de/SharedDocs/Downloads/DE/PDFPublikationen-DinA4/gruenbuch-arbeiten-vier-null. pdf?_blob=publicationFile\&v=2 (25.12.18)

BMBWF (Hrsg.) (2000): Lehrplan AHS Unterstufe. Geographie und Wirtschaftskunde. https:// bildung.bmbwf.gv.at/schulen/unterricht/lp/ahs9_784. pdf?61 ebyf (15.08.2018)

Budke, A. (2013): Einstiege. In: Rolfes, M. \& A. Uhlenwinkel (Hrsg.): Essays zur Didaktik der Geographie. o.S.

Ferrarÿ, A. (2014): 77 motivierende Unterrichtseinstiege für die Grundschule. Cornelsen Schulverlage, Berlin.

Klafki, W. (1963): Studien zur Bildungstheorie und Didaktik. Beltz Verlag, Weinheim/Bergstr. - Zitiert in: Ferrarÿ, A. (2014): 77 motivierende Unterrichtseinstiege für die Grundschule. Cornelsen Schulverlage, Berlin.

Klappacher, O. et al. (2014): geo-link 2. Lehr- und Arbeitsbuch. Geografie und Wirtschaftskunde für die 6. Schulstufe. Veritas Verlag, Linz.

Meyer, H. (2005): Unterrichtsmethoden. 2. Praxisband. Cornelsen Scriptor, Berlin.

Pichler, H. (2018): Einstiegstypen und Einstiegsmethoden ergänzt nach Budke, A. (2007): Einstiege in den Unterricht. In: Praxis Geographie 37(1). S 4-7, unveröffentlichte Lehrveranstaltungsunterlagen zum Begleitseminar (LV-Bezeichung) Sommersemester 2018. 


\section{Anhang: Schablonen}

Schablone 1: Uhr (dafür stärkeres Papier verwenden)

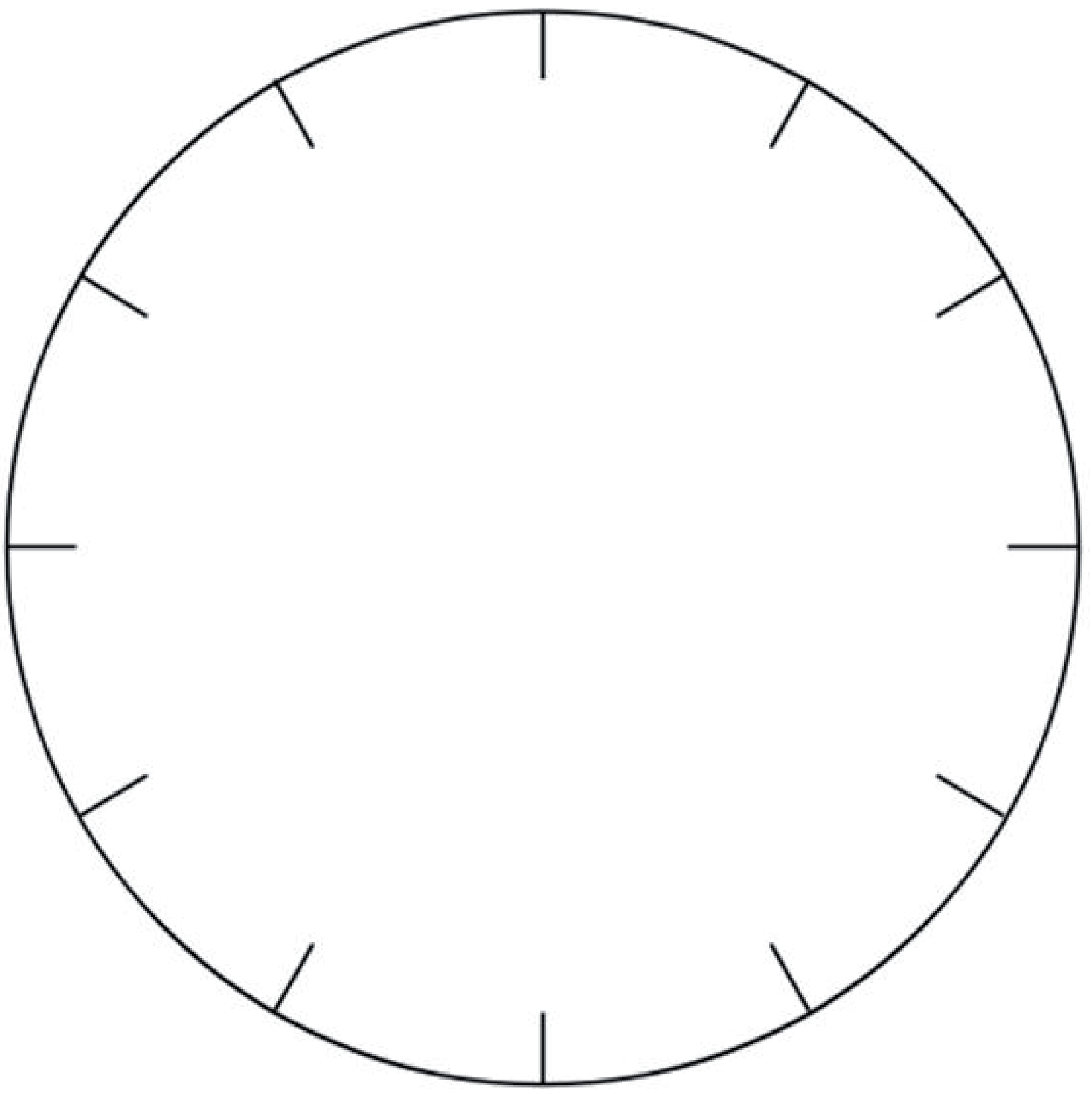




\section{Anhang: Schablonen}

Schablone 2: Minutenzeiger
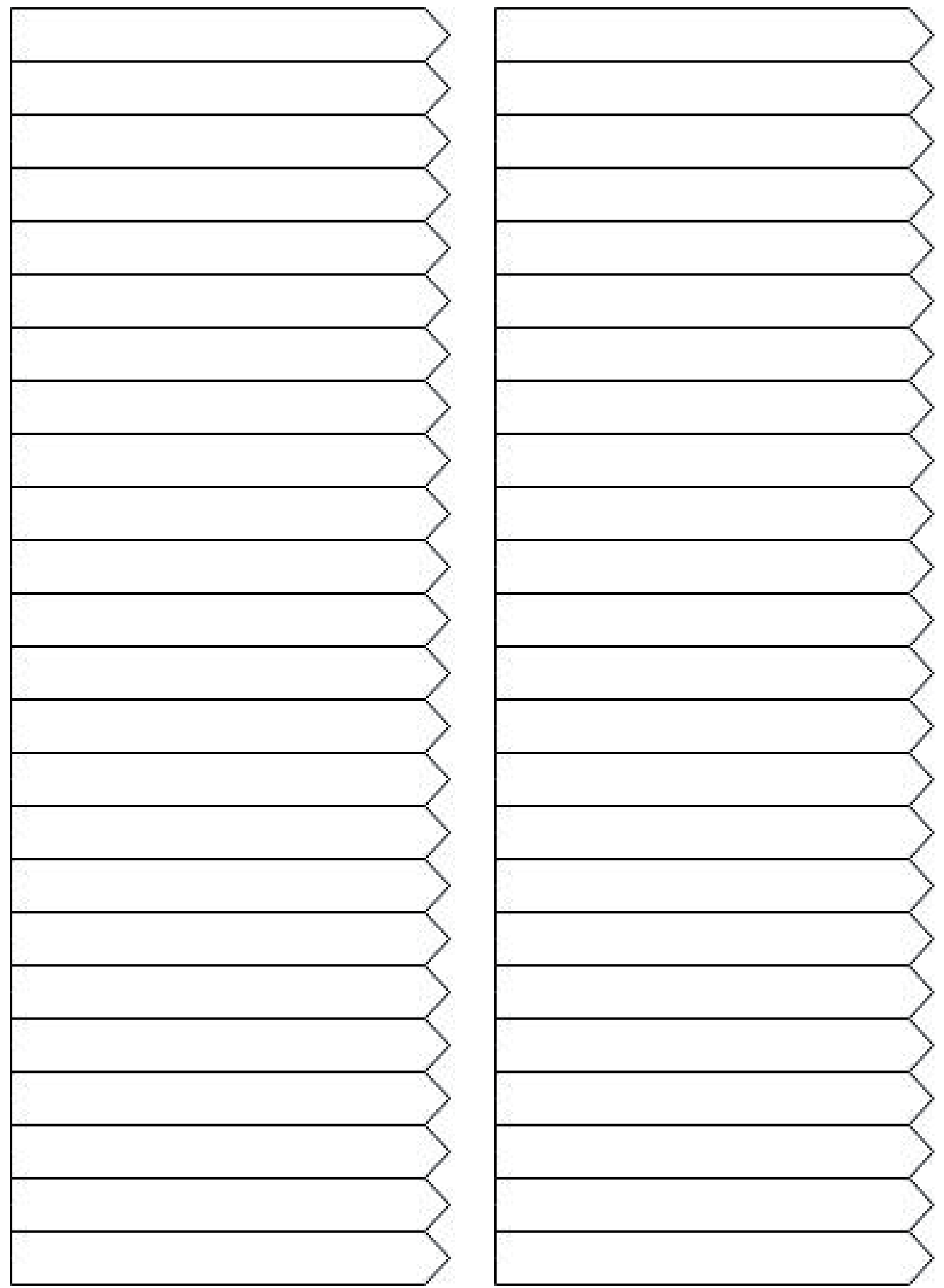
Anhang: Schablonen

Schablone 3: Stundenzeiger
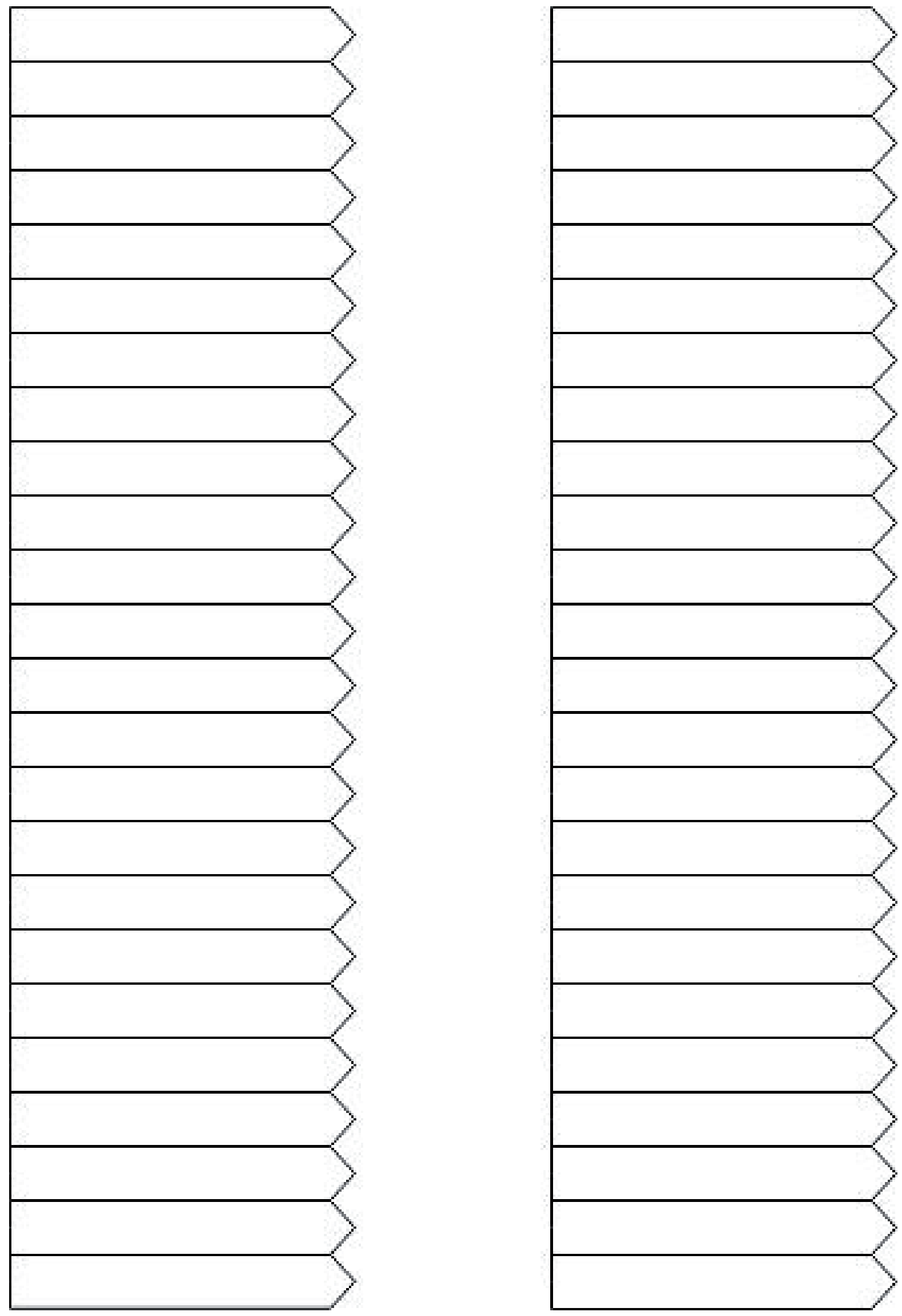